\title{
NON-LINEAR POISSON FUNCTION FOR NATURAL RUBBERS
}

\author{
Elli Gkouti ${ }^{1 *}$, Burak Yenigun ${ }^{1}, \mathrm{Krystof} \mathrm{Jankowski}^{2}$, Aleksander Czekanski ${ }^{1 * *}$ \\ ${ }^{1}$ Department of Mechanical Engineering, Lassonde School of Engineering, York University, Toronto, ON, Canada \\ ${ }^{2}$ Magna Closures of America Inc., Troy, MI, USA \\ *gkoutiel@yorku.ca,**alex.czekanski@lassonde.yorku.ca
}

\begin{abstract}
In linear elasticity, Poisson's ratio is one of the physical constants which characterizes an isotropic material, as it describes its lateral contractive response in infinitesimal strain. It has been proved that the use of this constant is limited, for several application where a material is subjected to large deformation. In this case, a scalar function of deformation can be defined, known as Poisson's function, but the strain tensor must be calculated first. Due to various existing strain tensors, several Poisson's functions are calculated to describe the negative strain tensor in the orthogonal direction, divided to the strain tensor in the axial direction, where the force is applied. Selected experimental tests are performed for elastomers under simple tension and the resulting Poisson's functions are shown for different strain tensors.
\end{abstract}

Keywords-rubber; Poisson function; non-linear; incompressible materials; elastomer;

\section{INTRODUCTION}

In classic isotropic, linear theory of elasticity, the mechanical behavior of a material can be described by determining only two constants: Young's modulus and Poisson's ratio. Any other information concerning the material's response, can be calculated by using the above constants, obtained from common experimental tests. Isotropic linear elasticity is capable of describing small deformations, where the lateral contractive response of a material is linearly proportional to the axial tension or compression [1]. Unfortunately, the above theory fails to describe large deformations that many applications and several materials, such as biological or elastomers, exhibit to. The reason is that the relations between stress and strain are more complicated and the material's parameters are not constant. As a result, the use of nonlinear elasticity is demanded due to its ability to describe the mechanical behavior as a scalar function of the deformation gradient. The complexity of defining such functions arise from the fact that there are multiple ways to define strains and stresses in nonlinear deformations, giving rise to multiple nonlinear functions corresponding to the same linear parameter. Furthermore, the choice of these functions is depended on how an experiment is performed and how the experimental data are processed [2].

\section{THEORETICAL BACKROUND}

\section{A. Non-Linear Elastic Deformation}

For an isotropic, non-linear hyperelastic material, the stress tensor can be described by a strain-energy density function $W=$ $W=W\left(I_{1}, I_{2}, I_{3}\right)$, which is expressed by the three invariants $I_{1}$ $(i=1,2,3)$ of Cauchy-Green deformation tensor $\mathbf{B}$. In the case of incompressible materials, namely $I_{3}=\lambda_{1} \lambda_{2} \lambda_{3}=1$, the constitutive equation becomes

$$
\boldsymbol{\sigma}=-p \mathbf{I}+\beta_{1} \mathbf{B}+\beta_{-1} \mathbf{B}^{-1},
$$

where $\mathbf{B}$ is the left Cauchy-Green tensor, which has the squared stretches $\lambda_{\mathrm{i}}{ }^{2}$ as principal values; $\boldsymbol{I}$ is the identity tensor, $p$ is the unknown hydrostatic stress and $\beta_{1}=2 \partial \mathrm{W} / \partial I_{1}, \beta_{2}=-2 \partial \mathrm{W} / \partial I_{2}$, are two response functions depending on the stress invariants, determined by experiments [3]. In the present work, the case of simple tension in the first direction is studied, which can be expresses by Batra's theorem [4], as $\sigma=\operatorname{diag}(T, 0,0)$, where $\operatorname{diag}(T, 0,0)$ refers to a diagonal matrix, and $T$ is the only nonzero component of the stress tensor. When a simple tension is applied, a corresponding extensional deformation is produced, in the form

$$
x_{1}=\lambda X_{1}, x_{2}=k(\lambda) X_{2}, x_{3}=k(\lambda) X_{3},
$$

where $\left(x_{1}, x_{2}, x_{3}\right)$ and $\left(X_{1}, X_{2}, X_{3}\right)$ are the Cartesian coordinates for the current and reference position, respectively; $\lambda$ is the extension ratio in the direction of where the force is applied and $k(\lambda)$ the stretch ratio in the orthogonal direction. The deformation gradient, the right and left Cauchy-Green tensors are

$$
\mathbf{F}=\operatorname{diag}(\lambda, k(\lambda), k(\lambda)), \quad \mathbf{C}=\mathbf{B}=\operatorname{diag}\left(\lambda^{2},[k(\lambda)]^{2},[k(\lambda)]^{2}\right) .
$$

For the special case of isochoric deformation, namely $\operatorname{det} \mathbf{F}=1$, the orthogonal stretch takes the form $k(\lambda)=\lambda^{-1 / 2}$ and the strain tensors becomes $\mathbf{e}_{\mathrm{n}}=\operatorname{diag}\left(\mathrm{e}_{\mathrm{n}}(\lambda), \mathrm{e}_{\mathrm{n}}(k(\lambda)), \mathrm{e}_{\mathrm{n}}(k(\lambda))\right)$, where the non-linear strain [2] is defined as 


$$
e_{n}(\mathrm{a})=\left\{\begin{array}{l}
\operatorname{lna}, \text { if } n=0, \\
\left(\mathrm{a}^{n}-1\right) / n, \text { if } n=0,
\end{array}\right.
$$

for any given stretch $\mathrm{a}>0$.

In Fig. 1, the values of different strain measures in the first direction appear, for several stretch ratios, when natural rubber is subjected to large deformation. Some of these tensors are commonly used, especially the ones that are independent of rotation, such as Hencky (logarithmic) strain tensor [5] $\mathrm{e}^{(\mathrm{H})}=\mathrm{e}_{0}$, the Biot strain tensor [6] $\mathrm{e}^{(\mathrm{B})}=\mathrm{e}_{1}$, the Green strain tensor [7] $\mathrm{e}^{(\mathrm{G})}=\mathrm{e}_{2}$, and the Almansi strain tensor [7] $\mathrm{e}^{(\mathrm{A})}=\mathrm{e}_{-2}$. The experimental data of a natural rubber, shown in Fig. 1, were compared with the different strain tensors, for examining their validity and the fitting was observed to be excellent. It must be noticed that, for small elastic deformations, the above strain tensors are equivalent to the infinitesimal strain from the linear elastic theory $\tilde{e}=\left(\nabla u+\nabla u^{\mathrm{T}}\right) / 2$, where the bar over a scalar or a tensor is used to denote a value appearing in the theory of linear elasticity [1]. Moreover, for small deformations, strain tensors are almost linear and equivalent to each other (Fig. 2).

\section{B. Non-Linear Poisson's Function}

In isotropic linear elasticity, Poisson's ratio describes the lateral contractive response of small deformations. When the deformation belongs to large scale, describing the mechanical behavior of the material with a constant, is not decent. As a result, Poisson's ratio becomes a scalar function of deformation [8], describing the negative quantity of strain in the orthogonal direction divided to the axial strain of the material in the direction where the force is applied. Due to the variety of the strain tensors in non-linear elasticity, Poisson function can be defined in several ways [9]. By using (4), the non-linear Poisson functions are defined as follows

$$
v_{\mathrm{n}}(\lambda)=-\mathrm{e}_{\mathrm{n}}(k(\lambda)) / \mathrm{e}_{\mathrm{n}}(\lambda) .
$$

The nonlinear Poisson function, defined by (5), can be calculated directly from experimental measurements. Consequently, no prior determination of the strain-energy density function is needed. For small deformations, i.e. when $\lambda \rightarrow 1$, the Poisson functions coincide with the Poisson's ratio from the linear elastic theory, namely $\tilde{\mathrm{v}}=\lim _{\lambda \rightarrow 1} v_{\mathrm{n}}(\lambda)=$ $\lim _{\lambda \rightarrow 1} \mathrm{~d} k(\lambda) / \mathrm{d} \lambda$. If a material is incompressible, then $k(\lambda)=\lambda^{-1 / 2}$. Usually, Poisson's ratio takes values between 0 and 0.5 [3], but for some materials it is noticed to be greater than 0.5. In Fig. 3, it is shown that Poisson functions can take similar values for finite deformations.

\section{NUMERICAL RESULTS AND DISCUSSION}

In order to check the validity of the theory presented above, some experiments took place concerning three different sample of natural rubber. The samples were exhibited to simple tension with $0.1 \mathrm{~mm} / \mathrm{s}$ speed, until they have reached their fracture point. The temperature that the experimental procedure took

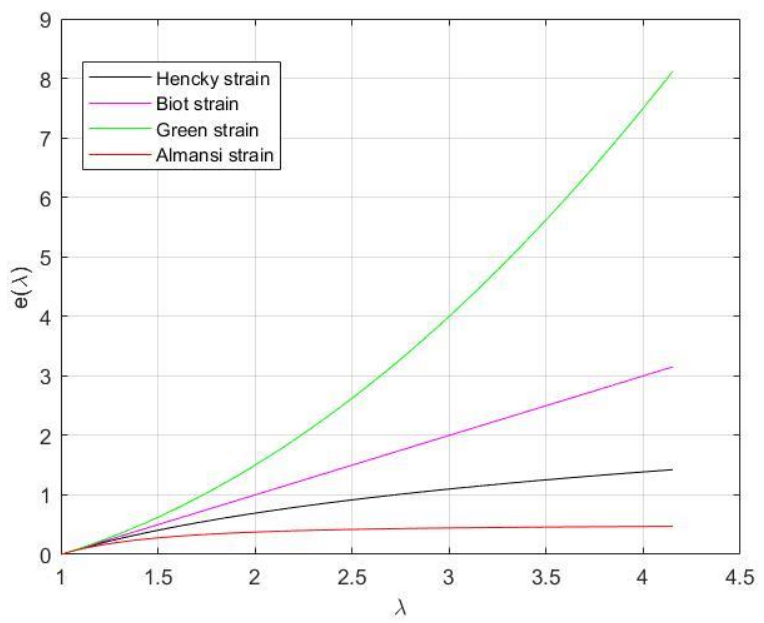

Figure 1. Strain tensors versus axial stretch ratio of a natural rubber for large deformation.

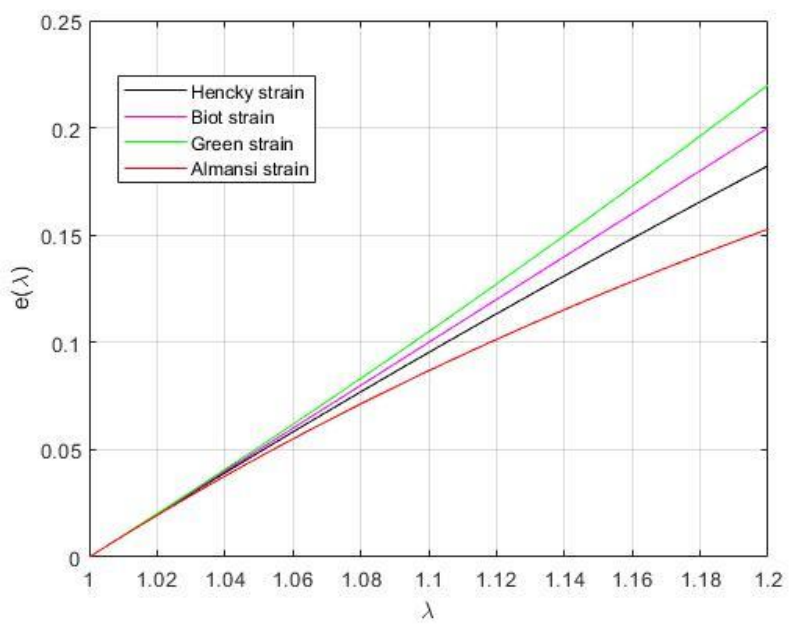

Figure 2. Strain tensors versus axial stretch ratio of a natural rubber for small deformation.

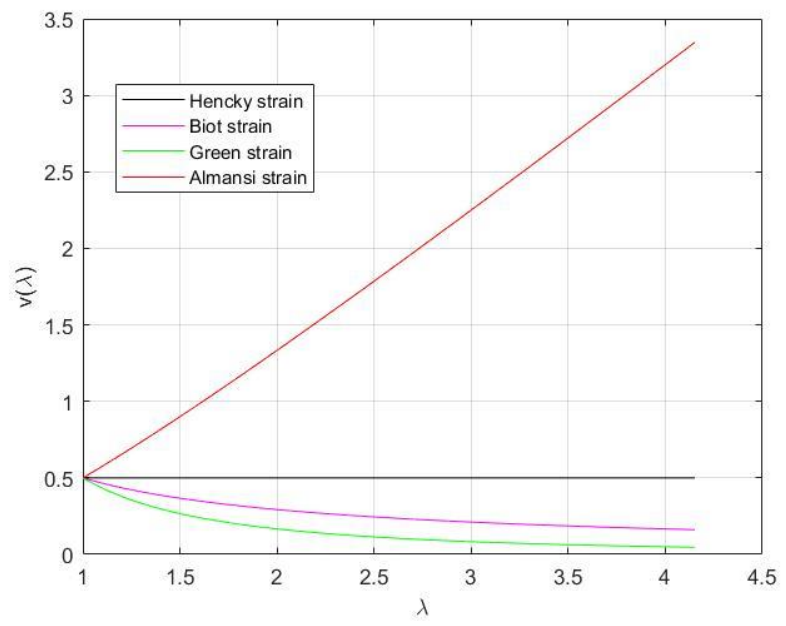

Figure 3. Poisson function versus axial stretch ratio for natural rubber. 
place was $23^{\circ} \mathrm{C}$. With the obtained experimental data, the lateral contractive response to stretch was examined for every sample. Different strain tensors where used in order to describe the extension of the material and the lateral response to it. The arising results shown in Fig. 1, were used for calculating Poisson functions for several values of axial stretch $\lambda$ to natural rubber (Fig. 3).

In Fig.1, it is observed that for small deformations the strain functions are linear and equal, but as the deformation grows the strain tensors describe the deformation of the material in different ways. For example, using the Hencky strain tensor, the description of the logarithmic strain is gained, but the Green strain tensor provides information about the material's deformation in respect to the reference configuration. In Fig. 3, different Poisson functions are shown for different strain tensors. It is observed that, for small deformations, the different Poisson functions are equal to the constant value of Poisson ratio, namely when $\lambda \rightarrow 1$ then $v \rightarrow 0.5$. The experimental results agree with the ones obtained by [9]. Moreover, it is significant that axial strain increases with the increasing axial stretch, but only by using the Hencky (logarithmic) strain the corresponding Poisson function remains constant and equal to 0.5. Namely, Poisson function using the Hencky strain tensor captures the remaining fixed volume, which is a significant characteristic property of natural rubber. In several applications, the use of constant material parameters is common when linear elastic models are used.

\section{CONCLUSIONS}

In the case of non-linear elasticity, scalar functions of the deformation must be defined, which are similar to the classical constitutive parameters. In the present work, natural rubbers are examined under simple tension for finite strain. The results show that the mechanical response of the material differs for large deformation and depends on the selected strain tensor. For small deformations, the strain tensors are almost linear and equal to each other, but when the stretch ratio is increased and so is the strain, the strain tensors differ. As a result, for small strain regime the Poisson's ratio is constant, but in finite strain, this ratio is converted to a scalar function of deformation.

\section{REFERENCES}

[1] A.E.H. Love, A Treatise on the Mathematical Theory of Elasticity, 4th ed., New York, NY: Dover, 1927.

[2] R. Hill, , "Constitutive inequalities for isotropic elastic solids under finite strain," Proc. Roy. Soc. London, vol. A314, pp. 457-472, 1970.

[3] C. Truesdell and W. Noll, The Non-Linear Fields Theories of Mechanics, Berlin, Germany: Springer-Verlag, 1965.

[4] R.C. Batra, "Deformation produced by a simple tensile load in an isotropic elastic body," Journal of Elasticity, vol. 6, pp. 109-111, 1976.

[5] H. Hencky, "Uber die form des elastizitatsgesetzes bei ideal elastischen stiffen," Z. Tech. Phys., vol. 9, pp. 215-220, 1928.

[6] M.A. Biot, Mechnanics of Incremental Deformationson, New York, London Sydney: John Wiley and Sons, Inc, 1965.

[7] R.W. Ogden, Ogden, Non-Linear Elastic Deformation, 2nd ed., New York, NY: Dover, 1997.

[8] M.F. Beatty and D.O. Stainake, "The Poisson Function of Finite Elasticity," Journal of Applied Mechanics, vol. 53, pp. 807-813, 1986.

[9] L.A. Mihai and A. Goriely, "How to characterize a nonlinear elastic material? A review on nonlinear constitutive parameters in isotropic finite elasticity," Proc. Roy. Soc. , vol. A473, pp. 20170607, 2017. 